\title{
Autonomous Multi copter Equipped with Robotic Arm for lifting Payloads
}

\author{
Raahul Varman ${ }^{1}$, Nageshwaran R R ${ }^{1}$, Nithyanand $U \mathrm{R}^{1}$, Franklin Telfer $\mathrm{L}^{2}$ and Brenda $\mathrm{M}^{2}$ \\ Student ${ }^{1}$, Associate Professor ${ }^{2}$, Department of Electronics and Communication Engineering, \\ Rajalakshmi Institute of Technology, Chennai
}

\begin{abstract}
The recent increase in the integration of Drones or UAV in civilian usage originate mainly from modern advancements in technology and the devices' abilities to accomplish civilian tasks faster, safe, and cost-efficient manner. The Applicative areas that witnessed tremendous UAV impact are Medical industry, Aerospace, Agriculture, Military, Online product Services and so on. Among several Applications, UAV technology is currently being implemented for delivery of products and medical derivative supplies. The main aim of this paper is to create a Prototype of drone interfaced with a robotic Arm for lifting payloads and to deliver them autonomously. The robotic arm is handled by gesture movements of the operator. The main advantage of this prototype is to increase the speed of transportation and by increasing its locomotive and design features we can lift payloads of higher mass to larger distances which is productive and useful for variety of fields given above.
\end{abstract}

\section{Keywords: UAV, Drones, Robotic arm}

\section{INTRODUCTION}

In the past few years, Unmanned Aerial Vehicles (UAVs) that are autonomously driven, often referred to as drones, have become a technology in renovating the future. Some of the classical acronyms for drones include: UAVs, UASs (Unmanned Aerial Systems), RPAs (Remotely Piloted Aircrafts) or ROAs (Remotely Operated Aircrafts). Due to the recent decrease in cost and improved performance of their corresponding subsystems, they have been showcased to the public for commercial buy. The idea of introducing

robotic hand in drone haven't reached on a commercial level and by creating an initial prototype, the challenges that forwards with this model can be challenging to resolve. But on the other side, the impact can be huge and beneficiary when it comes for medical resource transportation and Product delivery. But the common idea is to lift payload from the base station to the required destination.

\section{RELATED WORK}

[6] Due to delay of hospital aid transportation, many failures occur when the requirement doesn't reach on the fixed time. In order to save lives on time, a quadcopter is proposed here to deliver the requirements through air medium on time. This QC carries the blood derivatives to the required destination and it gets live tracked from the base station using a global positioning system (GPS) which is handled using an android application.

[5] The prototype exhibits their experimental solution for a minimal distance transportation of resources using quadcopters autonomously. A quadcopter enabled with autonomous flight is designed for delivering the orders from a 
processing unit. This quadcopter uses location maps to navigate to the required destination without any manual control. Using waypoints, the QC propagates from the base station to the destination point. Here the android device is interfaced with ArduPilot Master (APM) which is used as the flight controller module.

[1] Proposes the Robotic arm is being operated using Hand Gesture or the hand movements of the User or the operator. The Transmitter part uses Accelerometer from a Smart watch which basically uses hand movements and if android mobiles are used (or any iOS), then finger gestures are given as the necessary input. The transceivers connected to the device and the arm uses an android application to communicate which basically receives the commands through Bluetooth or Wi-Fi.

[7] The prototype developed here alerts the victim's condition to the doctors in the hospital and the ambulance using quadcopter technology. Where the quadcopter acts a mini patient monitoring embedded system, which can monitor victims heart rate, ECG values, temperature and respiration rate. he stated quadcopter acts as the fastest mode of transport in traffic congestion time. Where the quadcopter will reach the victims destination using GPS in a faster rate compare with ambulance.so he concluded, in future drone technology must be used in all important organization for better development.

\section{DESIGN METHOD}

This prototype model presented here is initially pursued to focus on industrial oriented payloads and medical emergency supplies and to place the requirement at the right time. In the case of emergency or demand of product, depending on the limitation over the quantity, the supply is picked up from the base station and the information from the client end regarding the destination which should be safer to land and at a communicable range without harming the surrounding or creating damage to the drone. Since its autonomous, GPS provides the coordinates for the drone to be directed and it is also used to monitor the drone at regular intervals. The robotic arm, on the other hand, using the gesture movements, lifts the payload and handles the package until it gets dropped at the required place. The research work and many other theories suggest that drones can reduce the time congestion.

\section{A. BLOCK DIAGRAM OF DRONE}

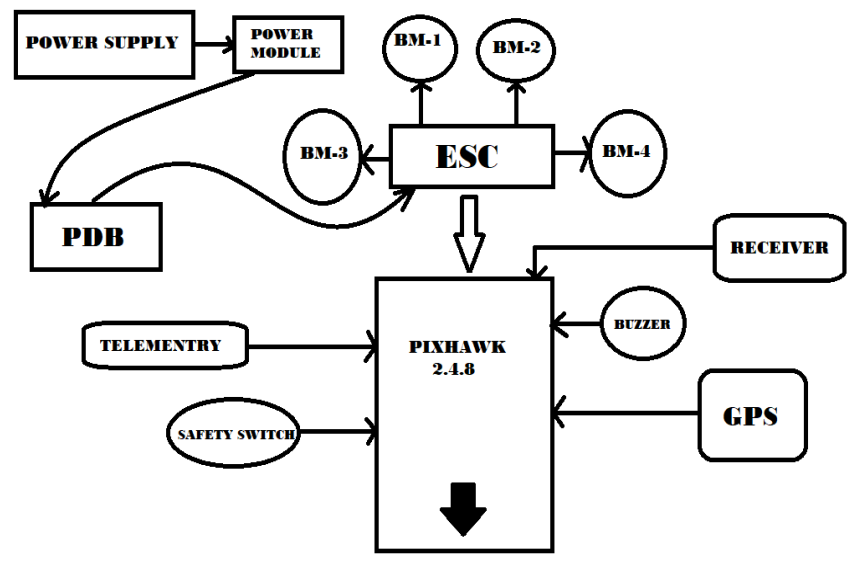

Fig 1

B. ROBOTIC ARM TRANSMITTER END

TRANSMITTER

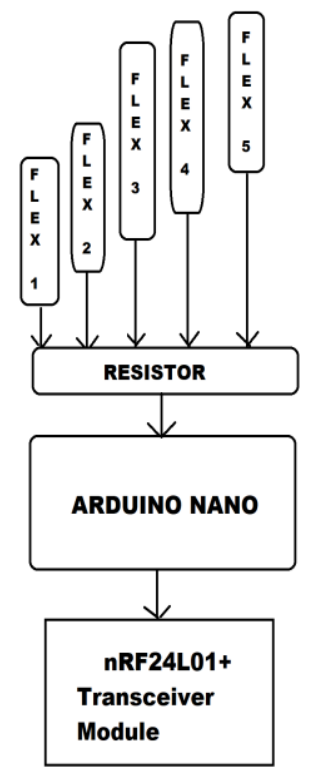


Fig 2

\section{ROBOTIC ARM RECIEVER END}

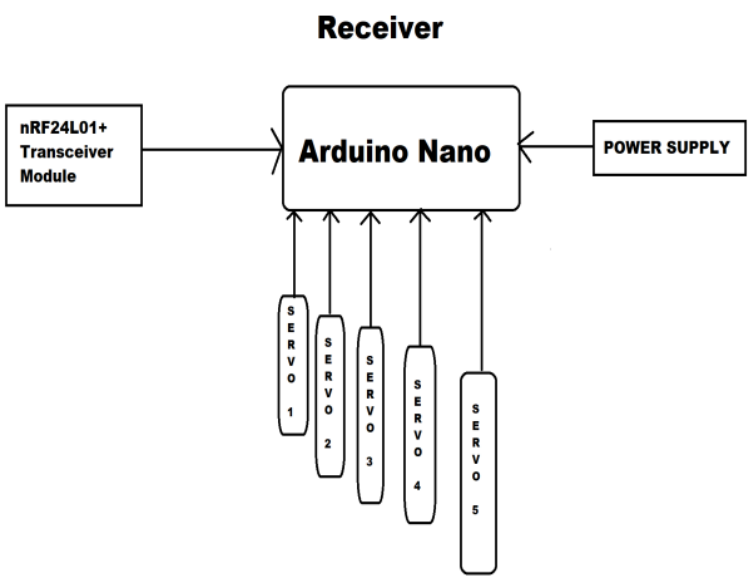

Fig 3

IV. EXPERIMENTAL SETUP

\section{A. Workflow of the system}

The workflow of the system begins from the user end where the client must provide prior information about the product that includes the type and quantity of the entire package and the destination point where the requirement must be delivered since the protype we devise here must support the range of transceiver used in the robotic arm to the range of distance the delivery occurs. Once it's compatible, then the package is ready for transportation. The block diagram shown in fig 1 shows the working model of our drone. The autonomous mission is carried out using an android application tower or qground control. Using that application, we create waypoints for the QC to travel to the end location. These waypoints are loaded to the flight controller through telementry. After setting the waypoints, arm the copter using the application and the QC takes off autonomously. Once the mission gets completed, the drone safely returns to the base location and it disables itself once it lands itself. The robotic arm connected to the drone lifts the payload from the home point using the operator's hand gestures and when the destination is reached, the arm drops the package from a safer distance to the ground. Fig 2 shows the transmitter end of the robotic arm interfaced to the drone and fig 3 shows the receiving end of the robotic arm which is interfaced to the drone.

\section{B. Hardware and software Technologies used}

\section{Hardware components}

\section{Quadcopter (QC) Frame (S500):}

The S500 frame is built from glass fiber giving it more toughness and durability. The arms have supporting ridges that gives the frame more stability. The main advantage of this frame is that the arms have an upsweep which helps in making the quadcopter stable. Another advantage is that the S500 avoids excess use of power distribution boards.

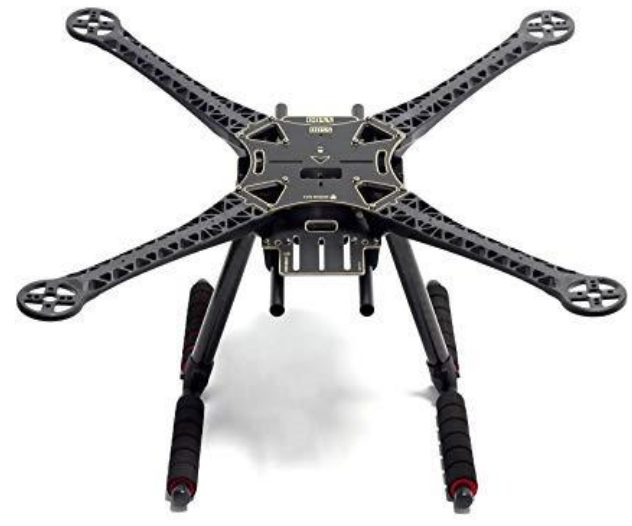

Fig 4

\section{Brushless Motor (BM):}

It is built using aluminium which accounts for its characteristics like small size, light weight and powerful. It has good quality magnets and well balanced for running setup smoothly. They are equipped with single strand wire for reliable connection with ESC. 


\section{Electronic Speed Controller (ESC):}

It has fast throttle response. The firmware of ESC is optimized and has good compatibility. Its features also include low resistance MOSFET and strong flow capacity. The voltage regulator IC which is supplied by the ESC is given to the microprocessor helping it acquire good antijamming capability. It has a safety electrical function which is necessary in case if power is turned on, it does not start the motor immediately without considering the throttle stick position. It avoids personal injury.

\section{Telementry:}

The radio telementry has air and ground modules separately. The transceivers can support both USB and UART connections. The telemetry shows live data on GPS position, heading, system voltage, waypoints. It has frequency modulation and spread spectrum (FHSS). It supports 2-way full duplex communication via adaptive time division modulation.

\section{Flux Sensors:}

It is simple to construct. It is $4.5 "$ in length. The flux sensors will bend and flex accordingly with the motion device. It follows the principle of angle displacement measurement. Fig 7 shows the flex sensors interfaced with Arduino nano with the telemetry for handling the robotic arm through gesture movements.

\section{GPS:}

It has a high level of robustness and flexible connectivity options. The advantages of M8N GPS are lower power consumption and higher precision. It also has anti jamming active $\mathrm{CW}$ detector. The M8N GPS has an in-built Odometer which gives information on the distance travelled.

\section{Arduino Nano:}

The Arduino Nano V3.0 is a small size and breadboard friendly. It used to as a platform for Programming. It acts as an interface to all components such as flux sensors and transceiver. It requires $6-12 \mathrm{~V}$ supply for the board to function. Fig 5 below shows the Arduino nano connected with the servo motors to handle the movements of the robotic arm.

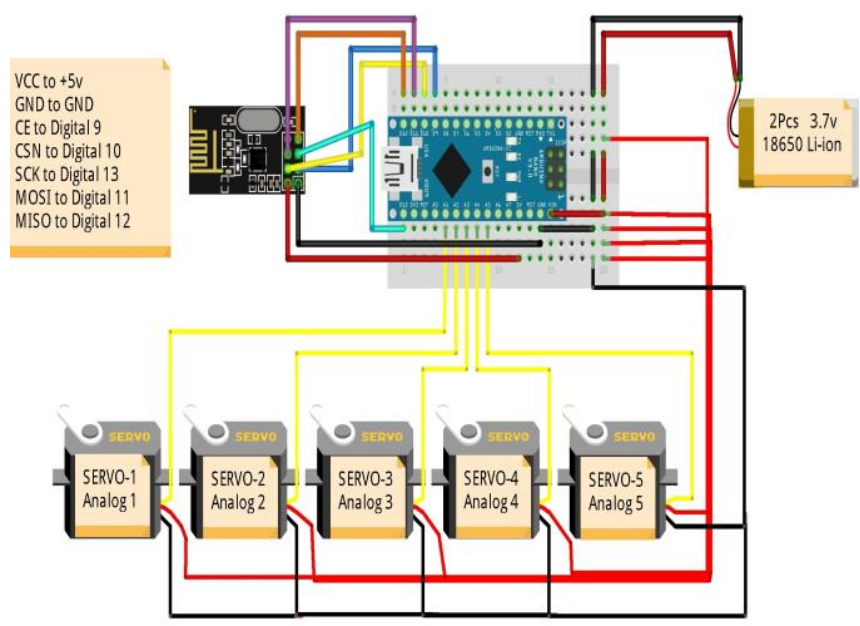

Fig 5 robotic arm receiving end

\section{Pixhawk Flight Controller:}

It is a rapidly evolving open source Ardupilot Mega. It supports waypoint-based navigation and two-way telemetry with radio telemetry modules. It contains $8 \mathrm{RC}$ channels with 4 serial ports. It supports both automatic and manual modes. It supports four bus interfaces: UART, CAN, I2C and SPI. It also provides buzzer interface. 


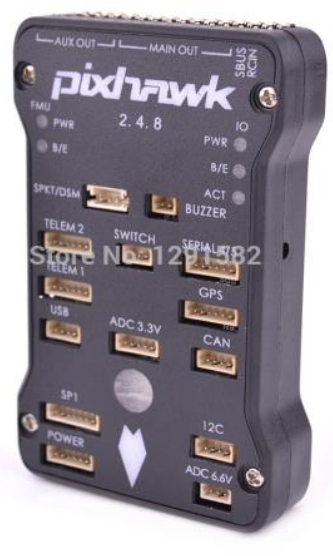

Fig 6 pixhawk flight controller

\section{Power Module:}

The power module provides supply for the flight controller. It gives information about the battery voltage and current. Using the information of battery voltage and current, the power consumption and performance of the battery can be determined. The power module is connected to the power distribution board (PDB) shown in fig 1 .

\section{0. nRF24L01 Transceiver:}

It is a wireless transceiver module that can send and receive data to robotic arm. The operating frequency of nRF24L01 is $2.4 \mathrm{GHz}$. It is legally used in all countries for engineering application purposes. The transmission of data speed is up to 2 megabits per second.

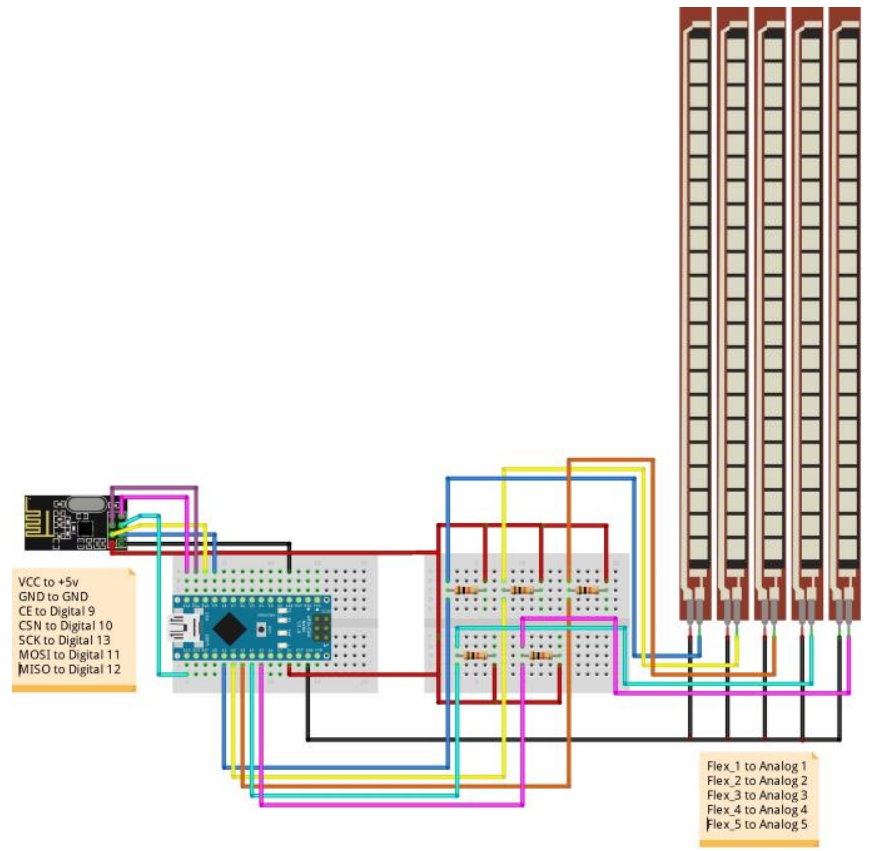

Fig 7 robotic arm transmitting end

\section{Software component}

\section{Machine planner:}

Mission Planner is a ground control station for Plane, Copter and Rover. It is compatible with Windows only. Mission Planner can be used as a configuration utility or as a dynamic control supplement for your

Autonomous vehicle. With appropriate telemetry hardware we can monitor the vehicle's status while in operation, Record telemetry logs which contain much more information the on-board autopilot logs, View and analyze the telemetry logs.

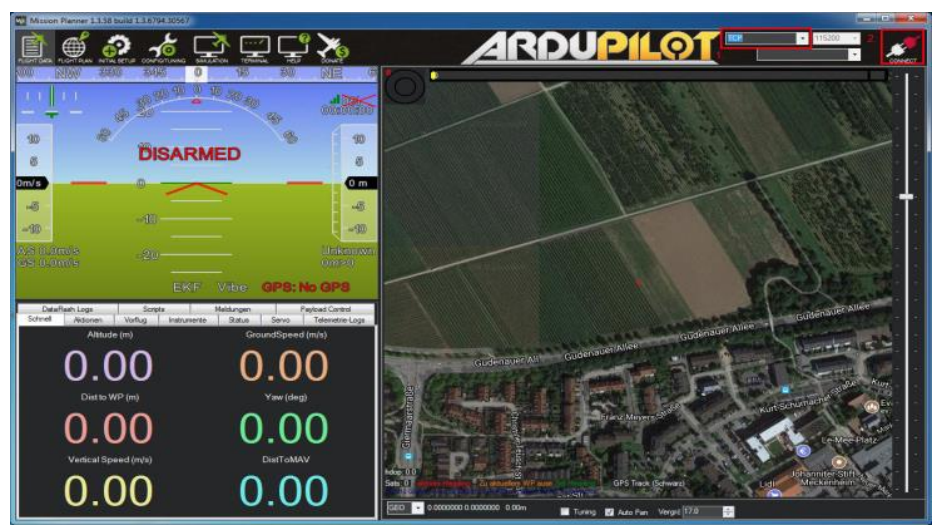




\section{Fig 8 machine planner opening window}

\section{PRACTICAL OBSERVATION}

The protype presented in this paper is taken into observation test to check the initial results about the takeoff of the quadcopter (QC), flight status, range of connectivity and the stability of drone after incorporating robotic arm and initiating the process by lifting a test payload. The test payload we used is of 150 grams and the flight test was stable and positive. The drone travelled at a height of 65 meters and travelled to a distance of 1.5 kilometers. The arm interaction with the payload during transportation is still under observation.

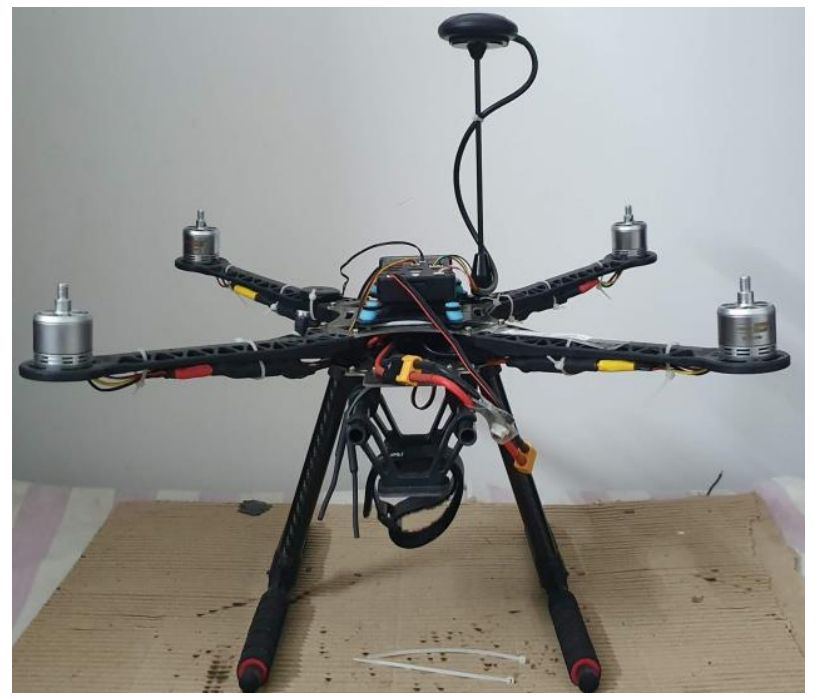

Fig 9 Practical drone setup

\section{CONCLUSION AND FUTURE WORKS}

The proposed system here is developed here on a prototype level to lift payloads on an industrial level and to carry the emergency aids in case of medical area. The base idea behind this drone equipped with robotic arm is to lift any kind of load from any location and drop them to the required destination and the initiative work is explained and the practical observation results is shown here. The future work of this drone is to lift larger mass which can be useful in saving lives by recovering them from places humans cannot enter. On an industrial basis, it can be used to operate high altitude workplaces and deep end areas by introducing machine learning algorithms and AI.

\section{REFERENCES}

[1] Bularka, S., Szabo, R., Otesteanu, M., \& Babaita, M. (2018). Robotic Arm Control with Hand Movement Gestures. 2018 41st International Conference on Telecommunications and Signal Processing (TSP).

[2] Sarker, P. P., Abedin, F., \& Shimim, F. N. (2017). R3Arm: Gesture controlled robotic arm for remote rescue operation. 2017 IEEE Region 10 Humanitarian Technology Conference (R10-HTC).

[3] Wu, X., Huang, Y., \& Kueh, S. (2016). Design and simulation of an aerial robotic arm. 2016 IEEE 11th Conference on Industrial Electronics and Applications (ICIEA).

[4] Yoo, H. D., \& Chankov, S. M. (2018). Drone-delivery Using Autonomous Mobility: An Innovative Approach to Future Last-mile Delivery Problems. 2018 IEEE International Conference on Industrial Engineering and Engineering Management (IEEM).

[5] Haque, M. R., Muhammad, M., Swarnaker, D., \& Arifuzzaman, M. (2014). Autonomous Quadcopter for product home delivery. 2014 International Conference on Electrical Engineering and Information \& Communication Technology

[6] Kumar R. Ashok ; P. Arulselvan; A. Ashif ; S. Gokul ; R. Kuppusamy(2019). Aero Ambulance Quad Copter Based Technology for An Emergency Healthcare. 2019 5th International Conference on Advanced Computing \& Communication Systems (ICACCS).

[7] Dhivya, A. J. A., \& Premkumar, J. (2017). Quadcopter based technology for an emergency healthcare. 2017 Third International Conference on Biosignals, Images and Instrumentation (ICBSII).

[8] Prutha Atre, Sahil Bhagat, NeVIL Pooniwala, Payal Shah. Efficient and Feasible Gesture Controlled Robotic Arm. 2018 SeCond INTERNATIONAL CONFERENCE ON INTELLIGENT COMPUTING AND CONTROL SYSTEMS (ICICCS) INSPEC ACCESSION NUMBER: 18510674 
[9] Ishac, K., \& Suzuki, K. (2016). Gesture based robotic arm control for meal time care using a wearable sensory jacket. 2016 IEEE International Symposium on Robotics and Intelligent Sensors (IRIS).

[10] Ackerman, E., \& Koziol, M. (2019). The blood is here: Zipline's medical delivery drones are changing the game in Rwanda. IEEE Spectrum, 56(5),

2431. doi:10.1109/mspec.2019.8701196

[11] Ackerman, E., \& Strickland, E. (2018). Medical delivery drones take flight in east africa. IEEE Spectrum,55(1),3435. doi:10.1109/mspec. 2018.

8241731

[12] Dayananda, K. R., Gomes, R., \& Straub, J. (2017). An interconnected architecture for an emergency medical response unmanned aerial system. 2017 IEEE/AIAA 36th Digital Avionics Systems Conference (DASC)

[13] Qi, J., Kang, J., \& Lu, X. (2017). Design and research of UAV autonomous grasping system. 2017 IEEE International Conference on Unmanned Systems (ICUS)

[14] Lan, G., Bu, Y., Liang, J., \& Hao, Q. (2016). Action synchronization between human and UAV robotic arms for remote operation. 2016 IEEE International Conference on Mechatronics and Automation. 